\title{
Therapeutic metformin/AMPK activation blocked lymphoma cell growth via inhibition of mTOR pathway and induction of autophagy
}

\author{
W-Y Shi ${ }^{1,3}$, D Xiao ${ }^{1,3}$, L Wang ${ }^{1,2}$, L-H Dong ${ }^{1}$, Z-X Yan ${ }^{1,2}$, Z-X Shen ${ }^{1}$, S-J Chen ${ }^{1,2}$, Y Chen ${ }^{* 1}$ and W-L Zhao ${ }^{*, 1,2}$
}

\begin{abstract}
Adenosine monophosphate-activated protein kinase (AMPK) acts as a major sensor of cellular energy status in cancers and is critically involved in cell sensitivity to anticancer agents. Here, we showed that AMPK was inactivated in lymphoma and related to the upregulation of the mammalian target of rapamycin (mTOR) pathway. AMPK activator metformin potentially inhibited the growth of B- and T-lymphoma cells. Strong antitumor effect was also observed on primary lymphoma cells while sparing normal hematopoiesis ex vivo. Metformin-induced AMPK activation was associated with the inhibition of the mTOR signaling without involving AKT. Moreover, lymphoma cell response to the chemotherapeutic agent doxorubicin and mTOR inhibitor temsirolimus was significantly enhanced when co-treated with metformin. Pharmacologic and molecular knock-down of AMPK attenuated metformin-mediated lymphoma cell growth inhibition and drug sensitization. In vivo, metformin induced AMPK activation, mTOR inhibition and remarkably blocked tumor growth in murine lymphoma xenografts. Of note, metformin was equally effective when given orally. Combined treatment of oral metformin with doxorubicin or temsirolimus triggered lymphoma cell autophagy and functioned more efficiently than either agent alone. Taken together, these data provided first evidence for the growth-inhibitory and drug-sensitizing effect of metformin on lymphoma. Selectively targeting mTOR pathway through AMPK activation may thus represent a promising new strategy to improve treatment of lymphoma patients.
\end{abstract}

Cell Death and Disease (2012) 3, e275; doi:10.1038/cddis.2012.13; published online 1 March 2012

Subject Category: Cancer

Incidence of lymphoma increases rapidly, ranking among the tenth most commonly diagnosed cancer. ${ }^{1}$ Relapsed from or resistant to conventional chemotherapy, prognosis of the patients, especially those with aggressive subtypes, remains un-optimal. New regimens need to be investigated, particularly those targeting signal transduction pathways on which malignant lymphoma cells depend for their survival.

Accumulating evidence has linked tumor progression to cellular metabolism, underscoring the possible therapeutic strategy of targeting metabolic pathways in cancers. ${ }^{2}$ Adenosine monophosphate-activated protein kinase (AMPK) is the main cellular energy sensor and actively participates in the interaction between metabolism and cancer. ${ }^{2}$ In normal lymphocytes, AMPK controls cell survival, mediates cell activation and metabolism. ${ }^{3,4}$ However, the role of AMPK in lymphoma is not clear. As mechanism of action, AMPK activation regulates tumor cell growth through the inhibition of the mammalian target of rapamycin (mTOR) pathway, which is generally involved in the control of translation initiation and protein synthesis. ${ }^{5}$ Also critical in lymphocyte development, ${ }^{6}$ mTOR is constitutively activated in various subtypes of lymphoma and contributes to tumorogenesis and drug resistance. $^{7-11}$ Whether therapeutic AMPK activation may target mTOR signaling and lymphoma progression remains great interest.

Metformin belongs to the biguanide class of oral hypoglycemic agents. Epidemiologic investigations reported that metformin treatment is associated with a decreased incidence of cancers like breast, ${ }^{12}$ prostate, ${ }^{13}$ colon $^{14}$ and pancreatic cancer. ${ }^{15}$ Experimental data confirmed the inhibitory effect of metformin on the growth of these tumor cells. ${ }^{16-18}$ Clinically, metformin improves outcome of diabetic cancer patients, either alone or combined with chemotherapy, indicating its potential role on cancer therapy. ${ }^{19,20}$ In hematological malignancies, recent update has demonstrated the activity of metformin against acute myeloid leukemia, because of AMPK-initiated mTOR inhibition. ${ }^{21}$ However, the efficacy of this clinically available AMPK activator has never been studied on lymphoma.

Autophagy is a cellular self-digestive process and of fundamental importance in tissue homeostasis. ${ }^{22}$ Although autophagy is primarily a protective process for the cell, it can also play a role in cell death. ${ }^{23}$ The mTOR pathway regulates cell autophagy under stress conditions, especially response to

\footnotetext{
${ }^{1}$ State Key Laboratory of Medical Genomics, Shanghai Institute of Hematology, Shanghai Rui Jin Hospital, Shanghai Jiao Tong University School of Medicine, Shanghai, China and ${ }^{2}$ Pôle de Recherches Sino-Français en Science du Vivant et Génomique, Laboratory of Molecular Pathology, Shanghai, China

${ }^{*}$ Corresponding authors: Y Chen and W-L Zhao, State Key Laboratory of Medical Genomics, Shanghai Institute of Hematology, Shanghai Rui Jin Hospital, 197 Rui Jin Er Road, Shanghai 200025, China. Tel: + 008621 64370045; Fax: + 008621 64743206; E-mails: rjchenyu @ tom.com (YC) or zhao.weili@yahoo.com (WLZ)

${ }^{3}$ These authors contributed equally to this work.

Keywords: lymphoma; adenosine monophosphate-activated kinase; mammalian target of rapamycin; metformin; drug sensitivity; autophagy

Abbreviations: AMPK, adenosine monophosphate-activated protein kinase; mTOR, mammalian target of rapamycin; p70S6K, p70 ribosomal S6 kinase; 4EBP1, 4E-binding protein 1 ; IC $\mathrm{I}_{50}$, Fifty percent of growth inhibition; PBS, phosphate-buffered saline; ACC, acetyl-CoA carboxylase; GAPDH, glyceraldehyde phosphate dehydrogenase; TUNEL, terminal deoxytransferase-catalyzed DNA nick-end labeling; IRS, immunoreactive score; SI, staining intensity

Received 26.9.11; revised 23.1.12; accepted 24.1.12; Edited by A Stephanou
} 


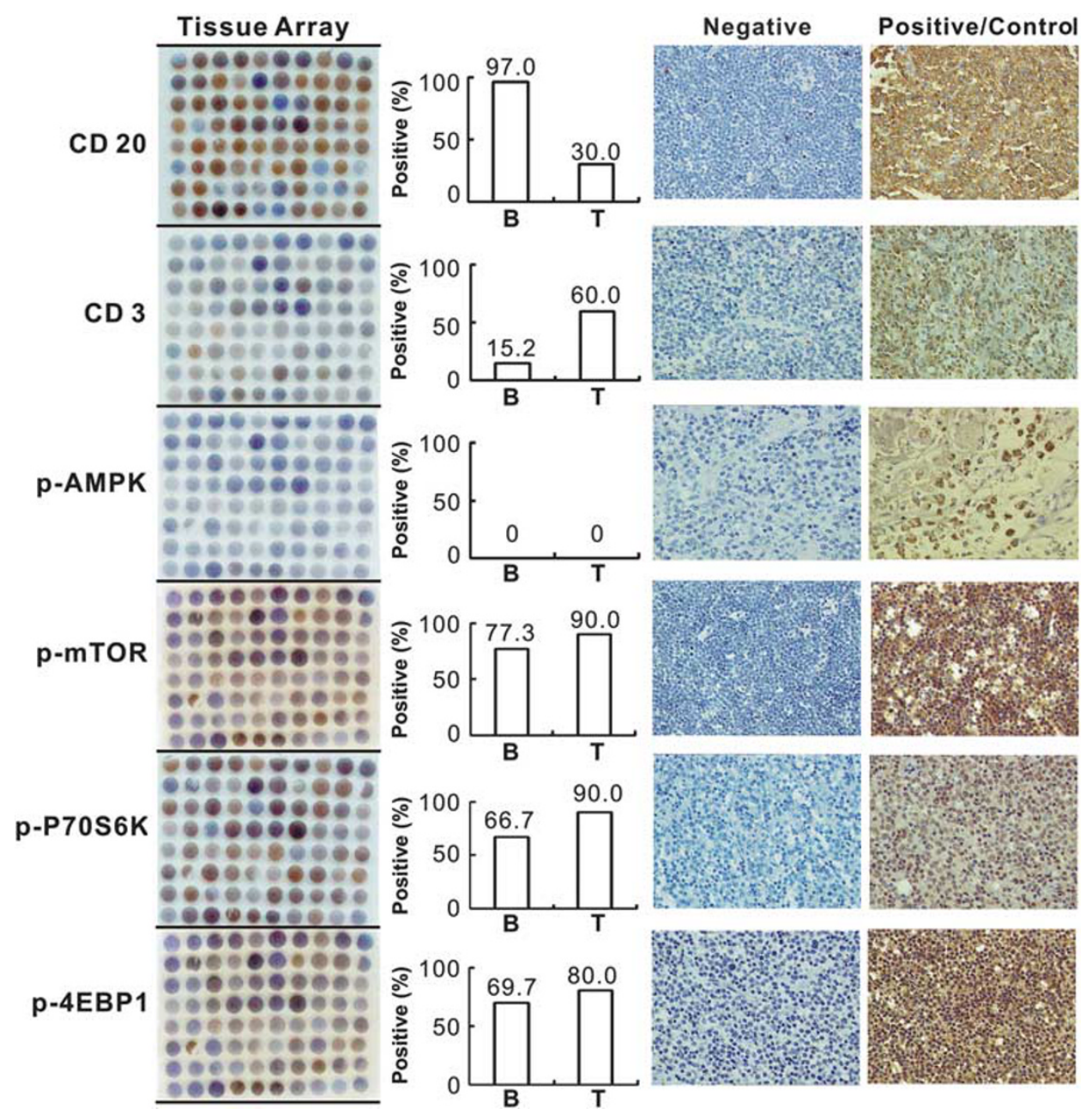

Figure 1 Dysregulation of AMPK/mTOR pathway in human lymphoma. Expression profile of phosphorylated form of AMPK, mTOR, as well as its downstream effectors p70S6K and 4EBP1 was assessed in human lymphoma tissue using tissue array. The melanoma tissue was served as the positive control of phosphorylated AMPK

anticancer agents. ${ }^{24}$ Defect in autophagy has recently been proposed to contribute to lymphoma progression, ${ }^{24,25}$ and, therefore, autophagy could be a promising target for lymphoma treatment.

In this study, we reported the potent antitumor action of AMPK activation in lymphoma. The AMPK activator metformin induced a dose-dependent suppression of lymphoma cell proliferation through negative control of the mTOR pathway. Importantly, lymphoma cell sensitivity to anticancer agents was enhanced by concomitant treatment of metformin via induction of autophagy. Our results provided first evidence for the potential of therapeutic metformin/AMPK activation as a novel approach for lymphoma treatment.

\section{Results}

The AMPK was inactivated in lymphoma and related to the upregulation of the mTOR signaling pathway. To examine the role of AMPK in human lymphoma, we first performed tissue array on primary tumor samples from 80 lymphoma patients (66 B-cell lymphoma, $10 \mathrm{~T}$-cell lymphoma and 4 Hodgkin's lymphoma), using antibodies against phosphorylated form of AMPK, mTOR and its downstream effectors p70 ribosomal S6 kinase (p70S6K) and 4E-binding protein 1 (4EBP1). As shown in Figure 1, the AMPK activity was completely lost in lymphoma cells. Consistent with the downregulation of AMPK expression, increased phosphorylation of mTOR, p70S6K and 4EBP1 were present in $77.3 \%, 66.7 \%$ and $69.7 \%$ of B-lymphoma cases, as well as $90.0 \%, 90.0 \%$ and $80.0 \%$ of T-lymphoma cases, respectively.

AMPK activator metformin inhibited lymphoma cell proliferation. Using MTT assay, we then determined the effect of AMPK activation on human B- and T-lymphoma cell lines. Fifty percent of growth inhibition $\left(\mathrm{IC}_{50}\right)$ was measured in these cells treated with AMPK activator metformin. Metformin exerted substantial growth inhibition in all lymphoma cell lines examined at concentrations ranging from 8.5 to $20.8 \mathrm{mM}$ (Figure 2a).

The dose-dependent response curves of two B cells (Daudi and SU-DHL-4) and two T cells (HUT78 and Jurkat) were shown in Figure $2 b$. Cell cycle was analyzed on these cells in 
a
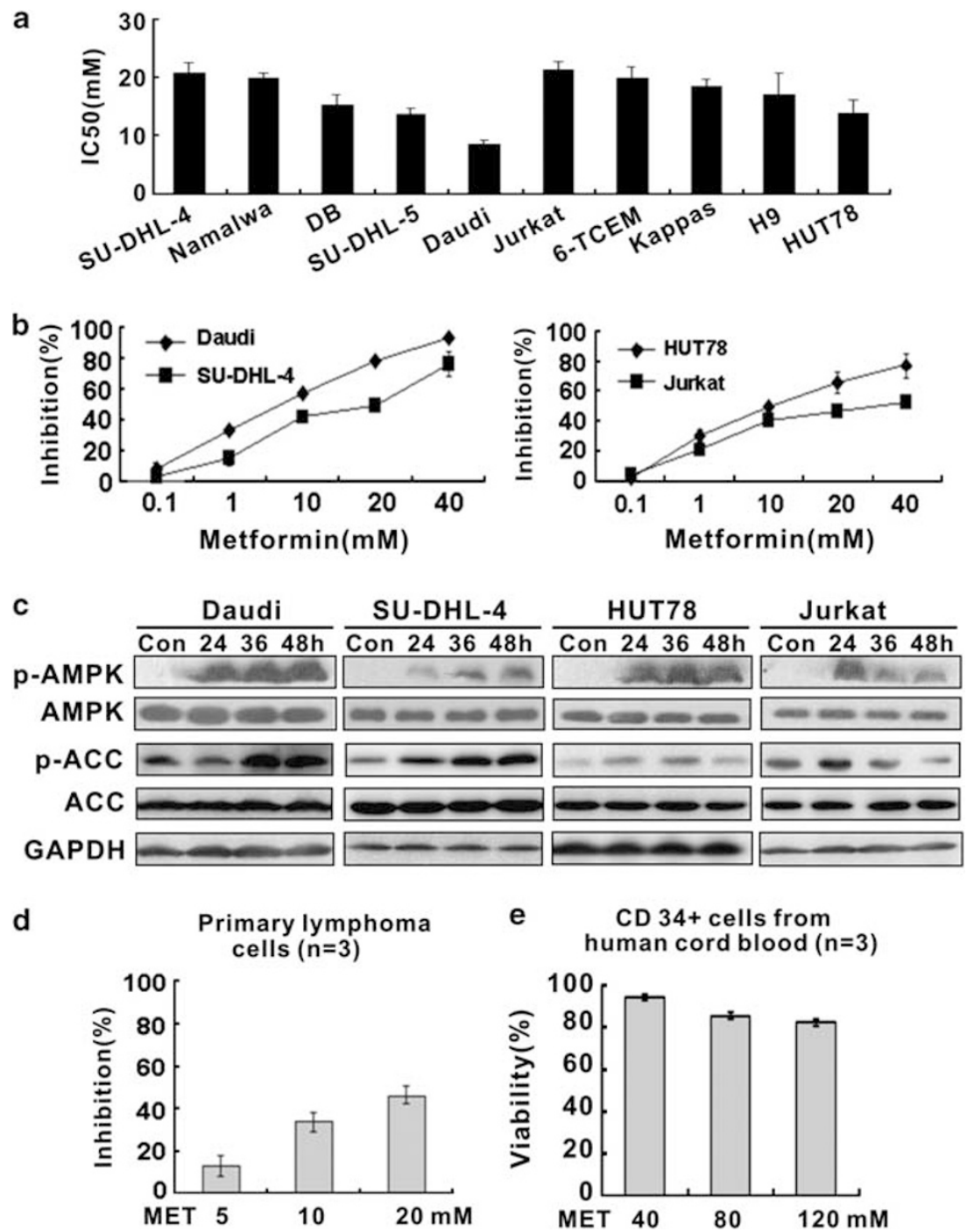

CD 34+ cells from
human cord blood $(n=3)$

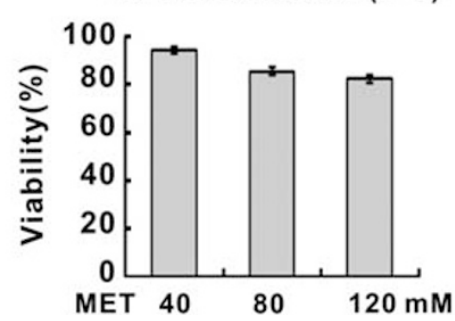

Figure 2 The inhibitory effect of metformin on growth of human lymphoma cell lines. (a) IC $C_{50}$ results obtained from MTT assay in a series of B- and T-lymphoma cell lines after metformin treatment. ( $b$ and $\mathbf{c}$ ) Response curves (b) and AMPK/ACC expression detected by western blot (c) in malignant B and T cells treated with metformin. (d) Metformin inhibited growth of primary lymphoma cells. (e) The proliferation of CD34 + cells enriched from cord blood samples was not altered by metformin up to $120 \mathrm{mM}$

the presence of metformin at $10 \mathrm{mM}$. Comparing with the control group $(32.6 \pm 1.0 \%$ for Daudi and $33.5 \pm 4.6 \%$ for SU-DHL-4 cells, respectively), the percentage of G0/G1 cells were significantly increased after metformin treatment for $36 \mathrm{~h}(54.6 \pm 2.3 \%$ for Daudi and $47.8 \pm 1.0 \%$ for SU-DHL-4 cells, $P<0.001$ and $P<0.01$, respectively). Similar results were also obtained in metformin-treated T-lymphoma cells (45.9 $\pm 5.9 \%$ versus $62.3 \pm 9.5 \%, P<0.05$, for HUT78 cells, and $44.5 \pm 1.9 \%$ versus $51.2 \pm 1.1 \%, P<0.01$, for Jurkat cells). Meanwhile, no significant elevation of annexin V-positive cells was found (data not shown). Western blot analysis revealed that metformin stimulated phosphorylation of AMPK and its downstream target acetyl-CoA carboxylase (ACC) (Figure 2c), indicating that the anti-proliferative effect of metformin on lymphoma cells involved AMPK activation.

In primary lymphoma cells, metformin resulted in significant growth inhibition from the concentration of $10 \mathrm{mM}$ (Figure $2 \mathrm{~d}$ ).
However, proliferation of CD34 + cells isolated from human cord blood, a population relatively enriched in hematopoietic progenitor cells, was not affected even at the concentrations up to $120 \mathrm{mM}$, suggesting that metformin exerted no major cytotoxic effect on normal hematopoietic precursors (Figure 2e).

Metformin modulated the AMPK/mTOR axis without involving AKT. On activation, AMPK inhibited the phosphorylation of mTOR, p70S6K and 4EBP1 (Figure 3a). To further determine if AMPK activation is responsible for the effect of metformin, we used the specific AMPK inhibitor compound $\mathrm{C}$ and the AMPK siRNA to block AMPK expression in Jurkat cells. Both pharmacologic and molecular knock-down of AMPK abrogated metformininduced lymphoma cell growth inhibition and cell cycle arrest (Figures $3 b$ and $c$ ). In addition to AMPK itself, AMPK 

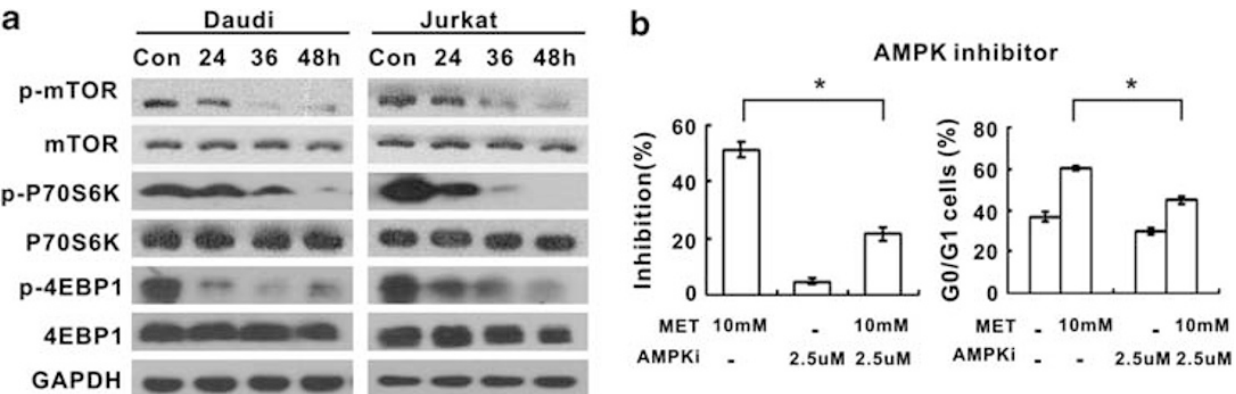

C
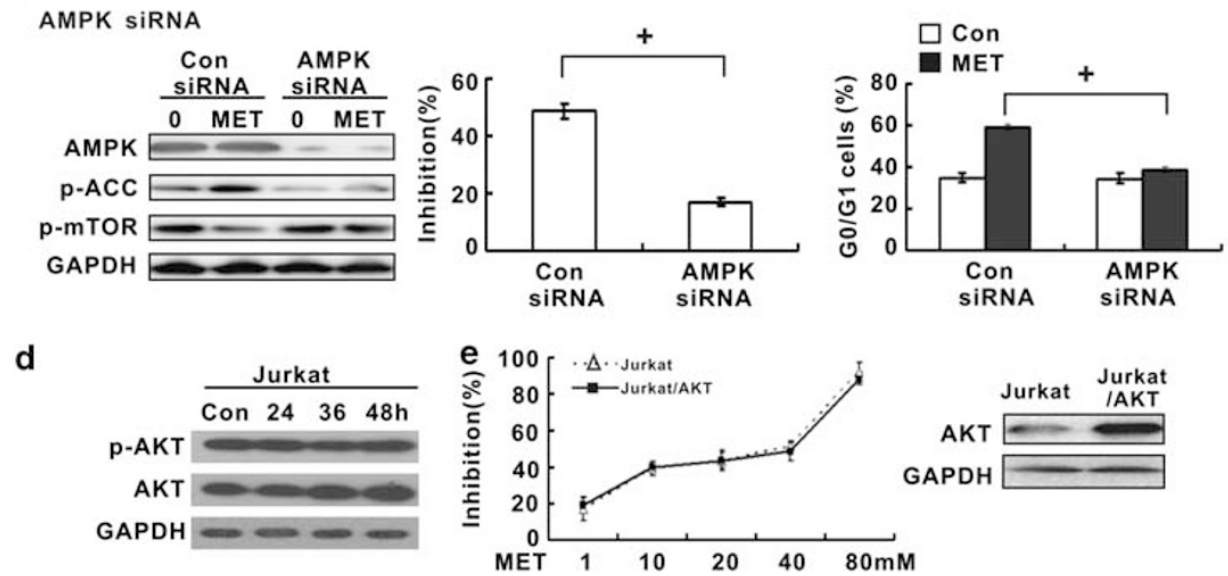

Figure 3 Metformin-induced mTOR inhibition in an AMPK-dependent manner. (a) Metformin decreased the expression of phosphorylated mTOR, as well as its effectors p70S6K and 4EBP1. (b and c) Specific AMPK inhibitor compound C (b) and AMPK siRNA (c) abrogated metformin-induced lymphoma cell growth inhibition and cell cycle arrest. Comparing with the negative control (Con siRNA), Jurkat cells transfected with AMPK- $\alpha$ siRNA (AMPK siRNA) failed to induce phosphorylated ACC and inhibit phosphorylated mTOR. ${ }^{*} P<0.001$ comparing with the metformin group. ${ }^{+} P<0.001$ comparing with the Con siRNA group. (d) The expression of phosphorylated AKT remained unchanged during metformin treatment in Jurkat cells. (e) Cell sensitivity to metformin was not affected in Jurkat cells transfected with AKT

silencing impaired the induction of ACC and inhibition of mTOR by metformin (Figure 3c). These results suggested that AMPK-initiated mTOR inhibition is essential for metformin to impart its growth-suppressive effect in lymphoma.

The mTOR inhibition may trigger a negative feedback loop, resulting in the AKT upregulation. ${ }^{26}$ When treated with metformin, expression of phosphorylated AKT remained constant (Figure 3d). Jurkat cells overexpressing AKT was also sensitive to metformin (Figure 3e). These data supported that metformin modulated AMPK/mTOR axis in an AKTindependent manner.

Metformin blocked in vivo lymphoma growth. The in vivo activity of metformin on lymphoma cells was also evaluated. Subcutaneous inoculation of Daudi or Jurkat cells into nude mice resulted in a tumor formation at the site of injection in all mice. In these murine xenograft models of $\mathrm{B}$ - and T-cell lymphoma, intraperitoneal treatment of metformin significantly inhibited tumor growth both in the low-dose and in the high-dose group (Figure 4a).

Decreased tumor size following metformin treatment was due to the reduced proliferation status of tumor cells as shown by Ki-67 staining on mice tumor sections. Terminal deoxytransferase-catalyzed DNA nick-end labeling (TUNEL) assay revealed no sign of lymphoma cell apoptosis. To search for more evidence of AMPK/mTOR involvement, immunohistochemical study of phosphorylated AMPK and mTOR were performed. As compared with the control group, phosphorylation of AMPK was remarkably increased, with that of mTOR decreased in the metformin group (Figure $4 \mathrm{~b}$ ).

Metformin acted together with the chemotherapeutic agent to inhibit lymphoma growth through induction of autophagy. Daudi and Jurkat cells were simultaneously treated with metformin and doxorubicin, a standard chemotherapeutic agent for lymphoma therapy. Isobolographic analysis yielded most of the data points within the envelope of additivity, denoting additive interactions in both cell lines (Figure 5a). AMPK knock-down by AMPK- $\alpha$ siRNA significantly decreased lymphoma cell response to metformin, alone or combined with doxorubicin (Figure 5b). Interestingly, expression of autophagy-related LC3-II was notably higher in the combination group than in the single-agent group at 72-h treatment (Figure 5c). The autophagy inhibitor 3-methyladenine significantly rescued lymphoma cells from the combined treatment, referring the growth-inhibitory effect as autophagy specific (Figure $5 \mathrm{~d}$ ).

To determine the chemo-sensitizing effect of metformin in vivo, we treated the xenograft models with metformin 
a

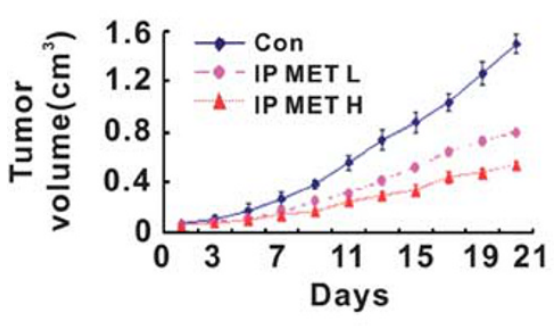

b

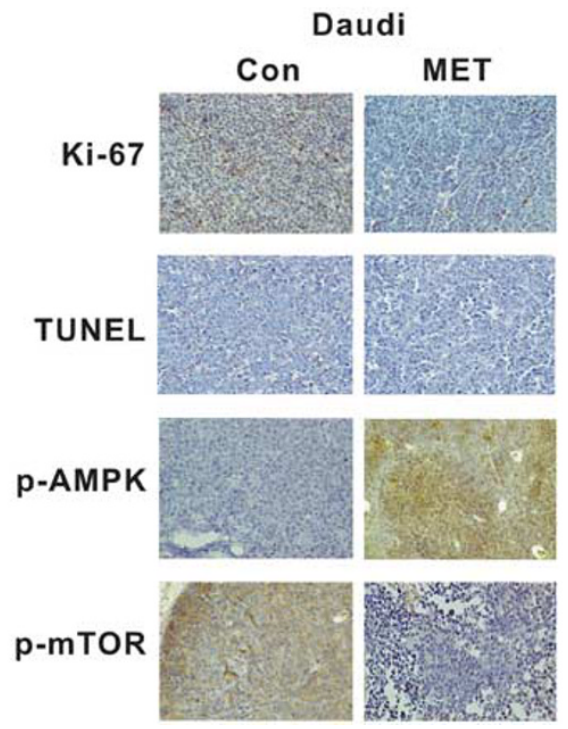

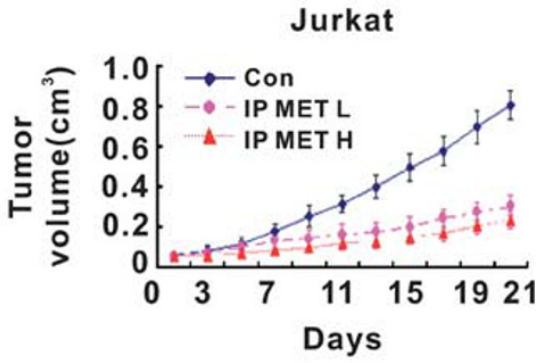

Jurkat

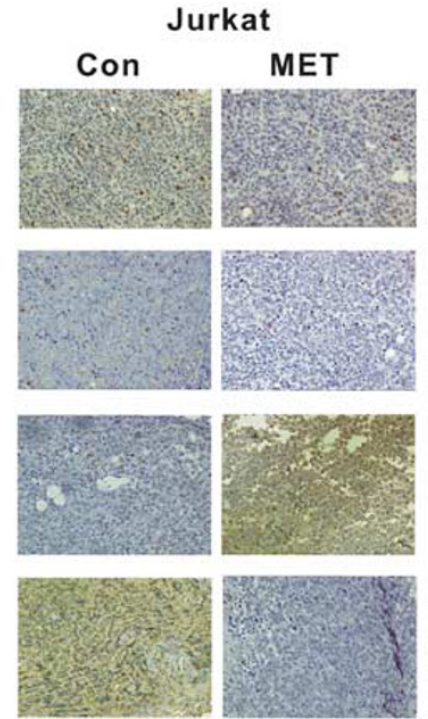

Figure 4 The effect of metformin in murine lymphoma xenograft models. (a) In both B-and T-cell lymphoma models, intraperitoneal treatment of metformin, both at the high-dose (IP MET H) and low-dose (IP MET L) group, significantly diminished xenograft tumor size. (b) As compared with the control group (Con), decreased proliferative index Ki-67 was identified, while TUNEL staining was negative in the metformin group (MET). Immunohistochemical study showed increased numbers of cells positive for phosphorylated AMPK, but decreased cells positive for phosphorylated mTOR in the metformin-treated tumors

and/or doxorubin. Considering that clinical use of metformin is typically performed by oral administration, and this would be a preferred treatment option, metformin was at this time given orally. As compared with either agent alone, dual treatment was clearly more effective on tumor growth (Figure 5e). Electron micrographic analysis showed that typical doublemembrane autophagic vesicles (autophagosomes) were most frequently identified in the combination group, further confirming the increase in autophagy (Figure 5f).

Metformin potentiated the effect of the mTOR inhibitor to induce lymphoma cell autophagy. The mTOR inhibitor has recently been used to treat lymphoma. ${ }^{11}$ We examined the interaction of metformin with clinical relevant mTOR inhibitor temsirolimus. In lymphoma cells, isobolographic analysis also revealed an additive effect (Figure 6a), which was significantly reduced in lymphoma cells transfected with AMPK- $\alpha$ siRNA (Figure 6b). The addition of metformin to temsirolimus at $72 \mathrm{~h}$ led to a remarkable increase in LC3-II expression (Figure 6c). 3-methyladenine abrogated the lymphoma cell growth inhibition, either induced by metformin or in combination with temsirolimus (Figure 6d). In vivo, the combined treatment of oral metformin and temsirolimus inhibited tumor growth and augmented cell autophagy in lymphoma-bearing mice, comparing with individual drug treatments (Figures $6 e$ and f).

\section{Discussion}

Metformin is the most widely used anti-diabetic drug in the world, and there is increasing evidence of a potential efficacy of this agent as an anticancer drug. ${ }^{27}$ Our study showed the activity of metformin against lymphoma both in vitro and in vivo. Importantly, metformin exerts a direct inhibitory effect on lymphoma cells with a minimal degree of toxicity against normal CD34 + hematopoietic precursors. Also the favorable therapeutic index was confirmed in murine lymphoma models without apparent systemic toxicity, indicating that metformin may be exploited for the management of lymphoma.

AMPK is a novel and biologically significant participant with a tumor-suppressor activity in the mitotic/cytokinetic phase of the cell cycle. ${ }^{28}$ The anti-lymphoma effect of metformin is AMPK dependent. In addition to elevated AMPK expression during metformin treatment, AMPK silencing using specific AMPK inhibitor and AMPK- $\alpha$ siRNA counteracted the metformin-induced inhibition of lymphoma cell growth and cell cycle progression. It is recently reported that the interplay between the AMPK- $\alpha$ subunit with the mitotic regulators in the centrosome and midbody coordinate the fundamental 
a

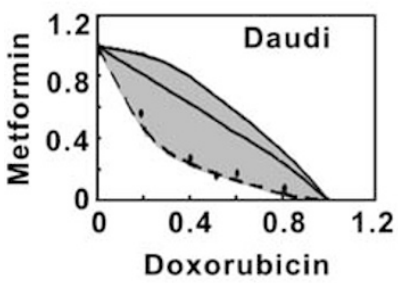

c

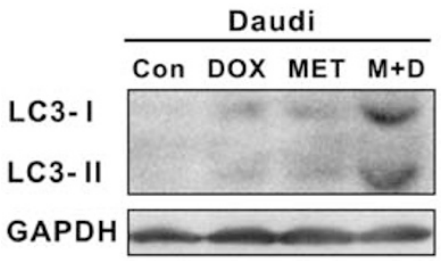

Isobologram

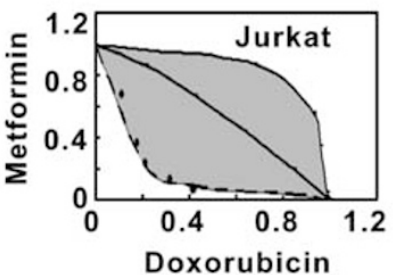

Doxorubicin

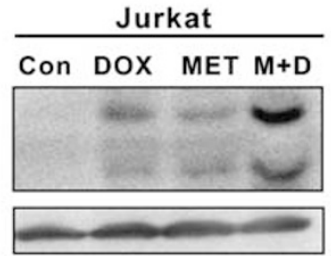

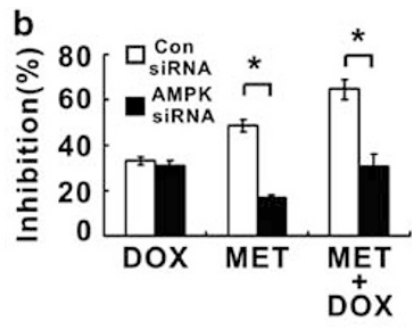

d Autophagy inhibitor

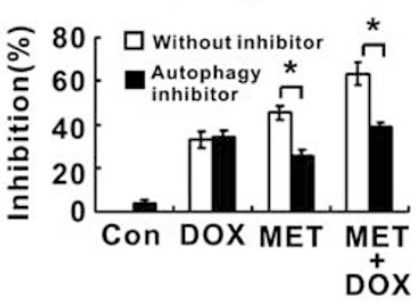

e

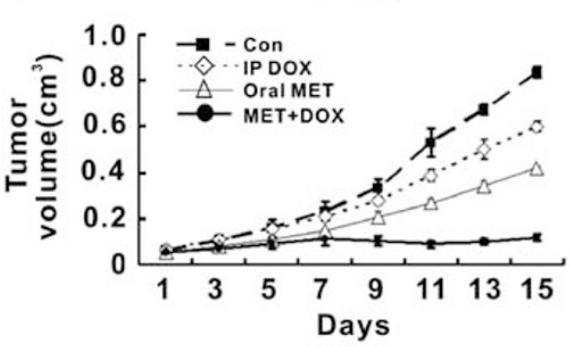

f

Daudi

Daudi

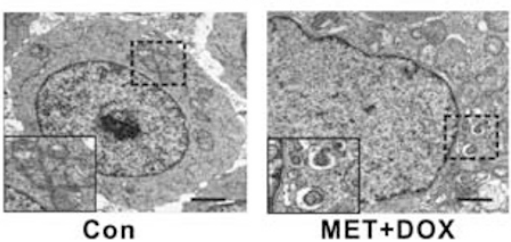

Jurkat
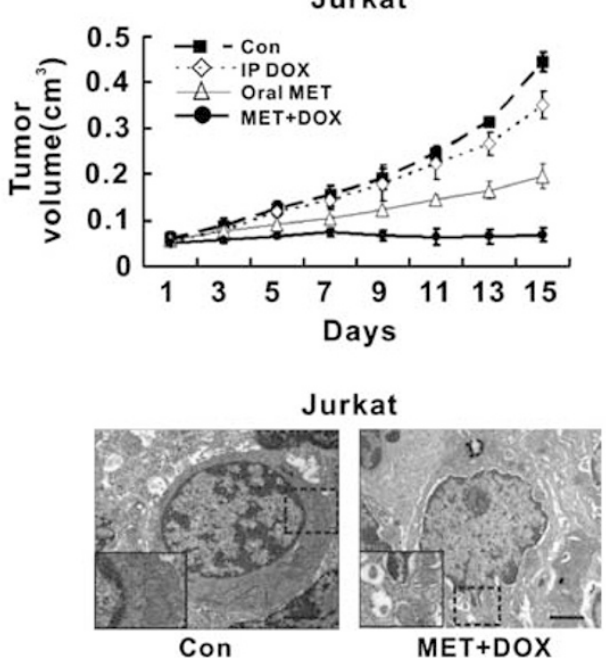

Figure 5 Metformin enhanced lymphoma cell sensitivity to the chemotherapeutic agent and stimulate autophagy. (a) In Daudi and Jurkat cells, metformin increased the cytotoxicity of the chemotherapeutic agent doxorubicin. (b) Metformin combined with doxorubicin induced less lymphoma cell growth inhibition in the AMPK siRNA group than in the Con siRNA group, ${ }^{\star} P<0.001$ comparing with the Con siRNA group. (c) Autophagy-related LC3-Il expression was significantly elevated in cells co-treated with metformin and doxorubicin. (d) Autophagy inhibitor 3-methyladenine abrogated lymphoma cell growth inhibition induced by co-treatment. (e) Combined oral metformin with doxorubicin significantly inhibited xenograft lymphoma growth, comparing with the control group or each agent alone. (f) The metformin/doxorubicin combination frequently induced lymphoma cell autophagy

biological process of genome division during mitosis and cytokinesis, and is closely related to the tumor-suppressive property of AMPK. ${ }^{28}$ More research is thus necessary to clarify the precise mechanism linking metformin-mediated AMPK activation to tumor cell cycle regulation.

Using tissue array, we confirmed that mTOR is consistently activated in lymphoma cells, as reported by other studies. ${ }^{7-11}$ AMPK-initiated mTOR inhibition is dispensable for the growthinhibitory action of metformin on lymphoma. On activation, the phosphorylated AMPK suppresses mTOR, as well as its downstream effectors p70S6K and 4EBP1, ultimately inhibiting cell growth. ${ }^{29}$ The mTOR inhibitors, although already been developed as anti-lymphoma therapies in clinical trials, are hampered because of the induction of multiple resistance mechanisms, particularly feedback activation of AKT. Moreover, recent study showed that rapamycin fails to block the expression of oncogenic proteins in acute myeloid leukemia, which is caused by the persistence of highly phosphorylated 4EBP1 molecules that maintain translation initiating complexes in active configuration in rapamycin-treated leukemia cells. ${ }^{21} \mathrm{New}$ approach should thus be considered to bypass these processes. Here, we found that metformin was not altered by AKT upregulation, and could significantly downregulate phosphorylated 4EBP1. Of note, metformin enhanced the lymphoma cell sensitivity to the mTOR inhibitor, further indicative of an important role of metformin on mTOR-activated lymphoma.

Metformin potentiated the effect of chemotherapy in lymphoma. Experimental data on the solid tumor showed that 
a

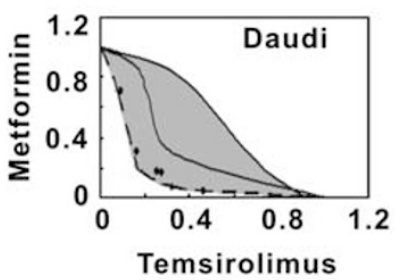

C
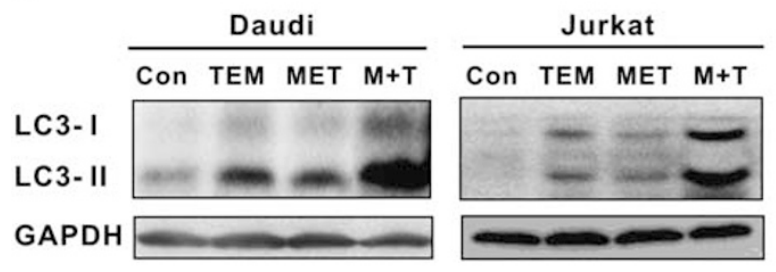

b

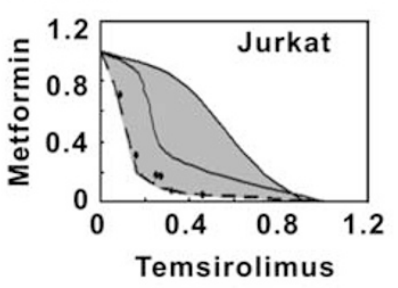

d Autophagy inhibitor
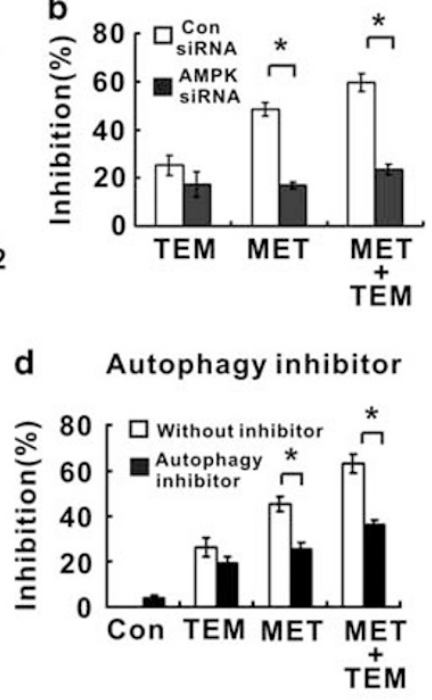

e

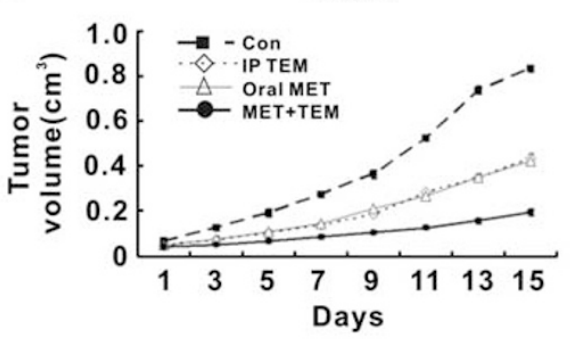

f

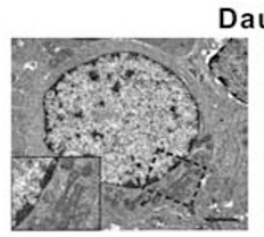

Con
Daudi

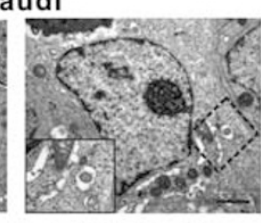

MET+TEM

Jurkat

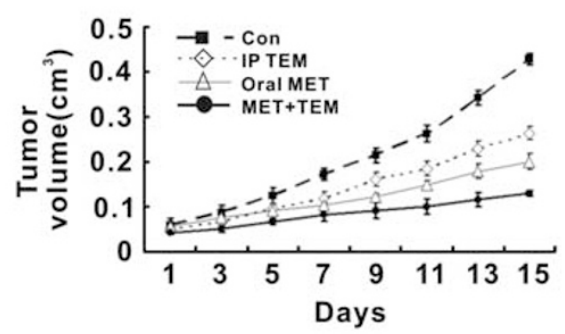

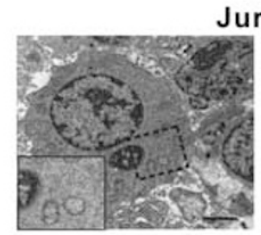

Con

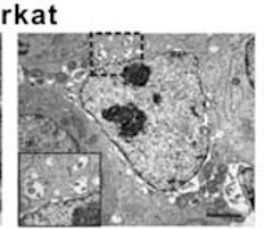

MET+TEM

Figure 6 Metformin potentiated the effect of mTOR inhibitor to induce lymphoma cell autophagy. (a) In Daudi and Jurkat cells, metformin enhanced the growth-inhibitory effect of the mTOR inhibitor temsirolimus. (b) Metformin combined with temsirolimus induced less lymphoma cell growth inhibition in the AMPK siRNA group than in the Con siRNA group, ${ }^{*} P<0.001$ comparing with the Con siRNA group. (c) Co-treatment resulted in LC3-II upregulation. (d) Lymphoma cell growth inhibition was overcome by autophagy inhibitor 3-methyladenine. (e) Combined oral metformin with temsirolimus significantly inhibited xenograft lymphoma growth, comparing with the control group or each agent alone. (f) The metformin/temsirolimus combination frequently induced lymphoma cell autophagy

metformin significantly inhibited proliferation of chemo-resistant cells, inducing cell cycle arrest accompanied by attenuated mTOR activation. ${ }^{30}$ Clinically, retrospective analyses suggested that metformin may improve patient response following neo-adjuvant chemotherapy for breast cancer. ${ }^{20}$ Our in vitro study demonstrated that metformin, beginning from a relatively lower dose $(1.25 \mathrm{mM})$, worked together with the chemotherapeutic agent in lymphoma. Correspondingly, in vivo tumor-inhibitory effect of doxorubicin was enhanced by metformin treatment. Oral metformin is equally effective, with the concentration used in xenograft experiments roughly comparable to what is used to treat human patients with type II diabetes. ${ }^{31}$ Based on the fact that the therapeutic advantage of oral metformin is related to its ability to selectively kill cancer stem cells, ${ }^{31}$ these findings further support the role of metformin in combination with chemotherapeutic agents that primarily target non-stem cancer cells.

Metformin targets tumor cells by different mechanisms. Not only it can amplify chemotherapy-induced AMPK activation and induces tumor cell apoptosis, ${ }^{32}$ but also inhibit tumor development through autophagy. ${ }^{33}$ Autophagy is an apoptosis-alternative pathway to induce cell death. Activation of autophagy improves the chemo-sensitizing effect of anticancer agents. ${ }^{34}$ In lymphoma, defect in autophagy correlates with a poor prognosis and less responsive to chemotherapy. ${ }^{25}$ Activation of AMPK could inhibit mTOR, the negative regulator of autophagy and then stimulates autophagy. ${ }^{35}$ Here, we found that metformin treatment enhanced tumor cell response to doxorubicin in murine lymphoma models in concert with increased autophagy in 
those tumors. This revealed a novel biological function for metformin to enhance the cytotoxic action of chemotherapeutic agents.

In conclusion, mTOR pathway dysregulation in lymphoma could be targeted by AMPK, providing a successful metabolism-regulating model for inhibition of tumor cell growth. Therapeutic metformin/AMPK activation, generally considered safe, well tolerated and remarkably inexpensive, offers great promise for lymphoma treatment.

\section{Materials and Methods \\ Cells. The B-lymphoma cell lines (SU-DHL-4, Nalmawa, DB, SU-DHL- 5 and Daudi) and T-lymphoma cell lines (Jurkat, 6T-CEM, Kappas, H9 and HUT78) are available from American Type Culture Collection (Bethesda, MD, USA). CD34 + cells were isolated from human cord blood using CD34 Progenitor Cell Isolation Kit (Miltenyi Biotech Inc., Auburn, CA, USA). Fresh lymphoma cells from the lymph node of three lymphoma cases were also obtained. Cells were maintained in RPMI- 1640 medium supplemented with $10 \%$ heat-inactivated fetal bovine serum in a humidified atmosphere of $95 \%$ air and $5 \% \mathrm{CO}_{2}$ at $37^{\circ} \mathrm{C}$. All biological studies were approved by the Institutional Review Board and informed consent was obtained in accordance with the Declaration of Helsinki.}

Reagents. Metformin (Sigma-Aldrich, St. Louis, MO, USA) was dissolved in phosphate-buffered saline (PBS) as a stock solution of $100 \mathrm{mM}$. Antibodies against phosphorylated ACC, ACC, phosphorylated AMPK, AMPK, phosphorylated mTOR, mTOR, phosphorylated p70S6K, p70S6K, phosphorylated 4EBP1, 4EBP1, phosphorylated AKT, AKT, LC3-II and chemiluminescence phototope-horseradish peroxidase kit were purchased from Cell Signaling (Beverly, MA, USA). Horseradish peroxidase-conjugated goat anti-mouse IgG and goat anti-rabbit IgG were from Santa Cruz Biotechnology (Santa Cruz, CA, USA). Anti-glyceraldehyde phosphate dehydrogenase (GAPDH) antibody was from Sigma-Aldrich. The AMPK inhibitor compound $C$ and the autophagy inhibitor 3-methyladenine were from Merck KGaA (Darmstadt, Germany).

Cell proliferation assay. Cells were seeded in 96-well plates and incubated in the presence of solvent control or the indicated concentrations of agents at $37^{\circ} \mathrm{C}$ for $72 \mathrm{~h}$. Cell proliferation was assessed by MTT assay and the absorbance was measured at $570 \mathrm{~nm}$ by spectrophotometry.

Flow cytometric assay. To assess the distribution of nuclear DNA content, cells were fixed overnight in $75 \%$ ethanol at $-20^{\circ} \mathrm{C}$, treated with $1 \%$ RNaseA for $15 \mathrm{~min}$ at $37^{\circ} \mathrm{C}$ and stained with $50 \mu \mathrm{g} / \mathrm{ml}$ propidium iodide. Cell apoptosis was analyzed using an ApoAlert Annexin V-FITC Apoptosis kit (Clontech, Palo Alto, CA, USA) according to the manufacturer's instructions. The fluorescent intensity was measured by flow cytometry.

Cell transfection. Jurkat cells were transfected with siRNAs targeting the AMPK- $\alpha 1$ and $\alpha 2$ subunit (Invitrogen, Life Technologies, Grand Island, NY, USA), or a control siRNA using AMAXA nucleofector Kit $\mathrm{V}$ following the manufacturer's instruction (AMAXA Biosystems, Cologne, Germany). Jurkat cells were also transfected with pcDNA3 Myr HA AKT1 (Addgene Inc., Cambridge, MA, USA) or a control vector pcDNA3, and electroporated at $200 \mathrm{~V} 25 \mathrm{~ms}$ in 4-mm cuvettes using a BTX ECM 830.

Western blot. Cells were lysed in $200 \mu$ lysis buffer $(0.5 \mathrm{M}$ Tris- $\mathrm{HCl}$, pH 6.8 , $2 \mathrm{mM}$ EDTA, $10 \%$ glycerol, $2 \%$ SDS and $5 \% \beta$-mercaptoethanol). Protein extracts $(20 \mu \mathrm{g})$ were electrophoresed on $10 \%$ SDS polyacrylamide gels and transferred to nitrocellulose membranes. Membranes were blocked with $5 \%$ non-fat dried milk in Trisbuffered saline and incubated for $2 \mathrm{~h}$ at room temperature with appropriate primary antibody, followed by horseradish peroxidase-conjugated secondary antibody. The immunocomplexes were visualized using chemiluminescence phototope-horseradish peroxidase kit. GAPDH was used to ensure equivalent protein loading.

Immunohistochemistry. Immunohistochemical analyses were carried out on 5- $\mu \mathrm{m}$ paraffin sections with an indirect immunoperoxidase method using antibodies against phosphorylated AMPK at $1: 100$ dilution, phosphorylated mTOR (Epitomics Inc., Burlingame, CA, USA) at 1:50 dilution, phosphorylated p70S6K at
1:60 dilution, phosphorylated 4EBP1 (Epitomics Inc.) at 1:100 dilution and Ki-67 (Dako, Glostrup, Denmark) at 1:100 dilution.

TUNEL assay. In situ cell apoptosis was confirmed by detection of fragmented DNA, using TUNEL assay, on 5- $\mu \mathrm{m}$ paraffin sections, using DeadEnd Colorimetric TUNEL System (Promega Corporation, Madison, WI, USA) according to the manufacturer's instruction.

Tissue array. A human lymphoma tissue array (LM802) was purchased from US Biomax, Inc. (Rockville, MD, USA) and analysis was conducted according to the manufacturer's suggested protocols. The melanoma tissue was served as the positive control for phosphorylated AMPK detection. Protein expression levels were scored semi-quantitatively based on staining intensity (SI) and distribution using the immunoreactive score (IRS). Briefly, IRS $=\mathrm{SI} \times \mathrm{PP}$ (percentage of positive cells). $\mathrm{SI}$ was determined as $0=$ negative; $1=$ weak; $2=$ moderate; and $3=$ strong. PP was defined as 1, <25\%; 2, 25-50\%; 3, 50-75\%; and 4, 75-100\% positive cells. IRS $\geq 4$ was referred as positive.

Transmission electron microscopy. Mice lymphoma samples were fixed overnight in $2 \%$ glutaraldehyde at $4{ }^{\circ} \mathrm{C}$, rinsed with $0.1 \mathrm{M}$ cacodylate buffer, postfixed in $1 \%$ osmium tetroxide for $1 \mathrm{~h}$ at $4{ }^{\circ} \mathrm{C}$, dehydrated in graded ethanol and embedded in Epon812 (TAAB Laboratories, Berkshire, UK). Ultrathin sections were prepared, stained with uranyl acetate and lead citrate, and examined on PhilipsCM120 transmission electron microscope (Eindhoven, The Netherlands). Ultrastructural studies were focused on double membrane-bound autophagic vesicles named autophagosomes, a long-established analytic gold standard for autophagy.

Tumor models. Two murine xenograft lymphoma models were applied to test the in vivo efficiency of metformin. Briefly, nude mice (5 to 6 weeks old, Shanghai Laboratory Animal Center, Shanghai, China) were injected into the right flank with $1 \times 10^{7}$ Daudi cells (mice received $300 \mathrm{cGy}$ for lymphoma cell engraftment) or $4 \times 10^{7}$ Jurkat cells, all of which developed tumors in 2 weeks. Treatments (six mice per group) were started after tumor became about $0.5 \mathrm{~cm} \times 0.5 \mathrm{~cm}$ in surface (day 0). For intraperitoneal use, the mice were randomly treated with PBS, low-dose $(2 \mathrm{mg} / \mathrm{kg} / \mathrm{day})$ or high-dose metformin ( $4 \mathrm{mg} / \mathrm{kg} / \mathrm{day})$ for 21 days. For oral use, the mice were randomly treated for 2 weeks with PBS, oral metformin $(3 \mathrm{mg} / \mathrm{kg} /$ day), intraperitoneal doxorubicin ( $3 \mathrm{mg} / \mathrm{kg} /$ day, twice weekly), intraperitoneal temsirolimus $(5 \mathrm{mg} / \mathrm{kg} /$ day every other day) or the combination treatment, respectively. Tumor volumes were calculated as $0.5 \times a \times b^{2}$, where ' $a$ ' is the length and ' $b$ ' is the width. All the mice experiments were performed in accordance with Institutional animal care and use committee procedures and guidelines.

Isobolographic analysis. Determination of the synergistic versus additive versus antagonistic-inhibitory effects of treatment combination was made using the isobologram of Steel and Peckham. On the basis of dose-response curves of the two drugs, three isoeffect curves were constructed. The area surrounded by the isoeffect curves was referred as the envelope of additivity. When the data points of the treatment combination fell within the envelope, the combination was regarded as additive.

Statistical analysis. Assays were set up in triplicates and the results were presented as mean \pm S.D. Variance between the experimental groups were determined by two-tailed $t$-test. $P<0.05$ was considered statistically significant.

\section{Conflict of Interest}

The authors declare no conflict of interest.

Acknowledgements. This work was supported, in part, by the National Natural Science Foundation of China (81172254), the Shanghai Commission of Science and Technology (11JC1407300) and the 'Shu Guang' project supported by Shanghai Municipal Education Commission and Shanghai Education Development Foundation (09SG21).

1. Jemal A, Siegel R, Xu J, Ward E. Cancer statistics, 2010. CA Cancer J Clin 60; 5: 277-300.

2. Cairns RA, Harris IS, Mak TW. Regulation of cancer cell metabolism. Nat Rev Cancer 2011; 11: 85-95. 
3. Cao Y, Li H, Liu H, Zheng C, Ji H, Liu X. The serine/threonine kinase LKB1 controls thymocyte survival through regulation of AMPK activation and $\mathrm{Bcl}-\mathrm{XL}$ expression. Cell Res 2010; 20: 99-108.

4. Maclver NJ, Blagih J, Saucillo DC, Tonelli L, Griss T, Rathmell JC et al. The liver kinase B1 is a central regulator of T cell development, activation, and metabolism. J Immunol 2011 187: 4187-4198.

5. Zoncu R, Efeyan A, Sabatini DM. mTOR: from growth signal integration to cancer, diabetes and ageing. Nat Rev Mol Cell Biol 2011; 12: 21-35.

6. Weichhart T, Saemann MD. The multiple facets of mTOR in immunity. Trends Immunol 2009; 30: 218-226.

7. Perez-Galan P, Dreyling M, Wiestner A. Mantle cell lymphoma: biology, pathogenesis, and the molecular basis of treatment in the genomic era. Blood 2011; 117: 26-38.

8. Yu BH, Zhou XY, Xiao XY, Yan SY, Qin T, Shi DR. Activation and clinicopathologic significance of AKT/mTOR signaling pathway in diffuse large B-cell lymphoma]. Zhonghua Bing Li Xue Za Zhi 2009; 38: 35-41.

9. Leseux L, Hamdi SM, Al Saati T, Capilla F, Recher C, Laurent G et al. Syk-dependen mTOR activation in follicular lymphoma cells. Blood 2006; 108: 4156-4162.

10. Vega $F$, Medeiros LJ, Leventaki V, Atwell $C$, Cho-Vega JH, Tian $L$ et al. Activation of mammalian target of rapamycin signaling pathway contributes to tumor cell survival in anaplastic lymphoma kinase-positive anaplastic large cell lymphoma. Cancer Res 2006; 66: 6589-6597.

11. Witzig TE, Gupta M. Signal transduction inhibitor therapy for lymphoma. Hematology Am Soc Hematol Educ Program 2010; 2010: 265-270.

12. Bodmer M, Meier C, Krahenbuhl S, Jick SS, Meier CR. Long-term metformin use is associated with decreased risk of breast cancer. Diabetes Care 2010; 33: 1304-1308.

13. Wright JL, Stanford JL. Metformin use and prostate cancer in Caucasian men: results from a population-based case-control study. Cancer Causes Control 2009; 20: 1617-1622.

14. Currie CJ, Poole CD, Gale EA. The influence of glucose-lowering therapies on cancer risk in type 2 diabetes. Diabetologia 2009; 52: 1766-1777.

15. Li D, Yeung SC, Hassan MM, Konopleva M, Abbruzzese JL. Antidiabetic therapies affect risk of pancreatic cancer. Gastroenterology 2009; 137: 482-488.

16. Dowling RJ, Zakikhani M, Fantus IG, Pollak M, Sonenberg N. Metformin inhibits mammalian target of rapamycin-dependent translation initiation in breast cancer cells Cancer Res 2007; 67: 10804-10812.

17. Zakikhani M, Dowling RJ, Sonenberg N, Pollak MN. The effects of adiponectin and metformin on prostate and colon neoplasia involve activation of AMP-activated protein kinase. Cancer Prev Res (Phila Pa) 2008; 1: 369-375.

18. Wang LW, Li ZS, Zou DW, Jin ZD, Gao J, Xu GM. Metformin induces apoptosis of pancreatic cancer cells. World J Gastroenterol 2008; 14: 7192-7198.

19. He XX, Tu SM, Lee MH, Yeung SC. Thiazolidinediones and metformin associated with improved survival of diabetic prostate cancer patients. Ann Oncol 2011; 22: 2640-2645.

20. Jiralerspong S, Palla SL, Giordano SH, Meric-Bernstam F, Liedtke C, Barnett CM et al. Metformin and pathologic complete responses to neoadjuvant chemotherapy in diabetic patients with breast cancer. J Clin Oncol 2009; 27: 3297-3302.

21. Green AS, Chapuis N, Trovati Maciel T, Willems L, Lambert M, Arnoult $C$ et al The LKB1/AMPK signaling pathway has tumor suppressor activity in acute myeloid leukemia through the repression of mTOR-dependent oncogenic mRNA translation. Blood 2010; 116: 4262-4273.

22. Mehrpour M, Esclatine A, Beau I, Codogno P. Overview of macroautophagy regulation in mammalian cells. Cell Res 2010; 20: 748-762.

23. Mizushima N, Levine B, Cuervo AM, Klionsky DJ. Autophagy fights disease through cellular self-digestion. Nature 2008; 451: 1069-1075.

24. Hanahan D, Weinberg RA. Hallmarks of cancer: the next generation. Cell 2011; 144 : 646-674.

25. Nicotra G, Mercalli F, Peracchio C, Castino R, Follo C, Valente G et al. Autophagy-active beclin-1 correlates with favourable clinical outcome in non-Hodgkin lymphomas. Mod Pathol 2010; 23: 937-950.

26. Wan X, Harkavy B, Shen N, Grohar P, Helman LJ. Rapamycin induces feedback activation of Akt signaling through an IGF-1R-dependent mechanism. Oncogene 2007; 26: 1932-1940.

27. Ben Sahra I, Le Marchand-Brustel Y, Tanti JF, Bost F. Metformin in cancer therapy: a new perspective for an old antidiabetic drug? Mol Cancer Ther 2010; 9: 1092-1099.

28. Vazquez-Martin A, Oliveras-Ferraros C, Lopez-Bonet E, Menendez JA. AMPK: evidence for an energy-sensing cytokinetic tumor suppressor. Cell Cycle 2009; 8: 3679-3683.

29. Wullschleger S, Loewith R, Hall MN. TOR signaling in growth and metabolism. Cell 2006; 124: $471-484$

30. Rattan R, Giri S, Hartmann L, Shridhar V. Metformin attenuates ovarian cancer cell growth in an AMP- kinase dispensable manner. J Cell Mol Med 2011; 15: 166-178.

31. Iliopoulos D, Hirsch HA, Struhl K. Metformin decreases the dose of chemotherapy for prolonging tumor remission in mouse xenografts involving multiple cancer cell types. Cancer Res 2011; 71: 3196-3201.

32. Rocha GZ, Dias MM, Ropelle ER, Osorio-Costa F, Rossato FA, Vercesi AE et al. Metformin amplifies chemotherapy-induced AMPK activation and antitumoral growth. Clin Cancer Res 2011; 17: 3993-4005

33. Tomic T, Botton T, Cerezo M, Robert G, Luciano F, Puissant A et al. Metformin inhibits melanoma development through autophagy and apoptosis mechanisms. Cell Death Dis 2011; 2: e199.

34. Lin Cl, Whang EE, Donner DB, Du J, Lorch J, He F et al. Autophagy induction with RAD001 enhances chemosensitivity and radiosensitivity through Met inhibition in papillary thyroid cancer. Mol Cancer Res 2010; 8: 1217-1226.

35. Viana R, Aguado C, Esteban I, Moreno D, Viollet B, Knecht E et al. Role of AMP-activated protein kinase in autophagy and proteasome function. Biochem Biophys Res Commun 2008; 369: 964-968.

Cell Death and Disease is an open-access journal published by Nature Publishing Group. This work is licensed under the Creative Commons Attribution-Noncommercial-No Derivative Works 3.0 Unported License. To view a copy of this license, visit http://creativecommons.org/licenses/by-nc-nd/3.0/ 